'Universidad de los Andes, Chile, Escuela de Enfermería.

2Escuela de enfermería, Pontificia Universidad Católica de Chile. Santiago, Chile.

${ }^{3}$ Clínica Universidad de los Andes. Chile.

${ }^{4}$ Center for Health Outcomes and Policy Research, School of Nursing, University of Pennsylvania, United States. aEnfermera.

bProfesor titular. CPhD.

Fuente de apoyo financiero: University of Pennsylvania Global Engagement Fund, Sigma ThetaTau International Global Nursing Research Grant (Linda Aiken, investigador principal).

Las autoras declaran no tener conflictos de interés.

Recibido el 31 de marzo de 2020 Aceptado el 21 de octubre de 2020.

Correspondencia a: Marta Simonetti

Dirección: Monseñor Álvaro del Portillo 12.455, Las Condes. Santiago, Chile. msimonetti@uandes.cl

\section{Dotaciones, skillmix e indicadores laborales de enfermería en Hospitales Públicos chilenos}

\author{
MARTA SIMONETTI ${ }^{1, \mathrm{a}, \mathrm{c}}$, PAZ SOTO ${ }^{2, \mathrm{a}, \mathrm{b}}$, \\ ALEJANDRA GALIANO ${ }^{3, a}$, MARÍA CONSUELO CERÓN ${ }^{1, a}$, \\ EILEEN T. LAKE ${ }^{4, a, c}$, LINDA H. AIKEN ${ }^{4, a, c}$
}

\section{Nurse staffing, skill mix and job outcomes in Chilean public hospitals}

Background: International evidence shows that there are organizational factors and nurse job outcomes that may negatively affect healthcare quality. Aim: To measure and analyze associations between nurse organizational factors, such as staffing ratios and skill mix, and job outcomes in public hospitals in Chile. Material and Methods: An observational, cross-sectional study of 1,855 registered nurses working in medical-surgical units in 37 public hospitals was conducted. Data collection followed the RN4CAST research protocol. Inferential analyses used logistic regression models. Results: The survey was answered by 1,395 registered nurses in 34 hospitals. The average staffing ratio was 14 patients-per-nurse, and the average skill mix was $31 \%$ registered nurses. Of all nurses, 35\% reported burnout, 22\% were dissatisfied, and 33\% intended to leave. Being burned out increased by 9 and $6 \%$ the odds of being dissatisfied and the intent to leave, respectively (Odds ratio $(O R) 1.09, p<0.01$ and 1.06, $p<0.01$ ). Being dissatisfied increased by five times the odds of intent to leave (OR 5.19, $p<0.01$ ). Conclusions: Staffing levels, burnout, and intent to leave warrant healthcare and governmental authorities' attention. All these factors may be threatening healthcare quality and safety.

(Rev Med Chile 2020; 148: 1444-1451)

Key words: Burnout, Psychological; Chile; Job Satisfaction; Personnel Turnover.
$\mathrm{E}$ n la última década, la inversión en salud del gobierno de Chile ha estado enfocada a mejorar la infraestructura hospitalaria y aumentar camas a través de la construcción de hospitales ${ }^{1}$. Este escenario pone de manifiesto la necesidad de tener recursos humanos adecuados para dotar estos hospitales. Potenciales amenazas para contar con suficiente personal de salud son la disponibilidad de profesionales y de recursos financieros para la generación de suficientes cargos.

Respecto de la disponibilidad de profesionales, desde 2016, el Ministerio de Salud de Chile (MIN-
SAL) viene estudiando las brechas de personal, aunque el análisis ha estado fuertemente enfocado en la necesidad de médicos ${ }^{2}$. Otros profesionales, como las enfermeras, han sido objeto de menor atención. Asimismo, los datos sobre los recursos financieros son poco alentadores. El gasto en salud y la deuda hospitalaria siguen aumentado, lo cual crea incentivos para bajar costos fijos como los de personal. Es común en la industria de salud que, frente a restricciones financieras, se recorte personal y, cuando cabe, se reemplace por personal menos capacitado y de menor costo ${ }^{3}$. 
Es importante prestar atención a las dotaciones de enfermeras ya que el cuidado de enfermería asegura la continuidad de atención y tiene relación con indicadores de calidad y seguridad. La investigación sobre los recursos de enfermería y su impacto en la calidad ha identificado dos tipos de factores que afectan los resultados de salud y la experiencia de los pacientes: los organizacionales y los individuales. Los factores organizacionales incluyen las dotaciones, o número de pacientes por enfermera, y el skillmix que mide la proporción de enfermeras en el total del personal de enfermería ${ }^{4,5}$. Los factores individuales, conceptualizados en la literatura como indicadores laborales de enfermería, incluyen el burnout, la insatisfacción y la intención de dejar el trabajo ${ }^{6}$.

Estudios internacionales muestran que dotaciones insuficientes y skillmix bajo impactan negativamente los resultados clínicos y la satisfacción usuaria $^{7-9}$. Lo mismo ocurre cuando las enfermeras sufren burnout e insatisfacción y tienen intención de dejar el trabajo ${ }^{10,11}$. Además de afectar a los pacientes, estos indicadores afectan el desempeño institucional generando ausentismo y rotación, con el consecuente aumento de $\operatorname{costos}^{12-14}$.

Los hospitales chilenos tienen una subdotación de enfermeras no por falta de profesionales en el mercado sino por falta de cargos en el sistema público $^{15}$. En 2012, el MINSAL puso a disposición de los hospitales recomendaciones de dotación basadas en la complejidad de las camas ${ }^{16}$. Dichas recomendaciones están lejos de la realidad de países de Norteamérica y Europa ${ }^{17}$. En parte, la brecha se produce por un desajuste entre la clasificación teórica de las camas y la complejidad real de los pacientes. El problema de la subdotación se agrava por la concentración de horas médicas en las mañanas, con déficit en la tarde y noche ${ }^{15}$. Esto lleva a que la atención de los pacientes, durante estas horas, dependa más críticamente del escaso personal de enfermería. Respecto del skillmix, no existen datos nacionales. Según las recomendaciones del MINSAL ${ }^{16}$, los hospitales que las cumplen debieran tener en unidades de cuidados básicos o medios de adultos un skillmix de alrededor de $40 \%$. En Europa, para unidades similares, la cifra promedio es cercana a $65 \%{ }^{5}$.

En cuanto a indicadores laborales, estudios locales han explorado burnout e insatisfacción entre las enfermeras chilenas ${ }^{18,19}$, pero no existe un análisis global. Datos como el de ausentismo laboral ameritan una mirada profunda a posibles determinantes. En los sectores de la administración pública, el de salud es el que presenta mayor número de días no trabajados por licencias médicas $^{20}$.

El propósito del siguiente estudio es medir y analizar asociaciones entre dotaciones, skillmix e indicadores laborales de enfermería en hospitales públicos de alta complejidad a nivel nacional. Este estudio se enmarca en la implementación del protocolo de investigación RN4CAST-Chile ${ }^{21,22}$, liderado por las Escuelas de Enfermería de la Universidad de Pennsylvania (Estados Unidos) y Universidad de los Andes (Chile) en colaboración con las Escuelas de Enfermería de la Pontificia Universidad Católica de Chile, Universidad Católica del Maule y Universidad de La Serena.

\section{Material y Método}

Estudio multicéntrico, observacional, de corte transversal. Aprobado por los Comités de Ética de la Universidad de los Andes y de los Servicios de Salud de las instituciones participantes.

Se trabajó con el universo de hospitales que cumpliesen con los siguientes criterios de inclusión: hospitales públicos de alta complejidad, de adultos, con más de 100 camas, y que a la fecha de inicio de recolección de datos contaran con reporte de IR-GRD $(\mathrm{N}=37)$. Cabe señalar que este último criterio es relevante en el contexto de toda la investigación, pero no para la presente publicación. Se excluyeron hospitales pediátricos o de especialidad. En cada hospital, se invitó a participar a todas las enfermeras que cumpliesen con los siguientes criterios de inclusión: trabajar en unidades médicas o quirúrgicas y prestar cuidado directo a pacientes $(\mathrm{N}=1.853)$. La participación, voluntaria y anónima, consistió en responder una encuesta, previa firma de consentimiento informado.

Las principales variables estudiadas fueron dotación, skillmix e indicadores laborales: burnout, insatisfacción laboral e intención de dejar el trabajo. La encuesta incluyó también preguntas sociodemográficas y sobre educación y experiencia profesional. La dotación se calculó como el número de pacientes presentes en el último turno dividido por el total de enfermeras en la unidad, según el reporte de las enfermeras encuestadas. 
Del reporte individual de las enfermeras de un mismo hospital, se sacó el promedio general de la dotación de dicho hospital. El skillmix se calculó dividiendo el total de enfermeras en la unidad por la suma de enfermeras, técnicos de nivel superior en enfermería y auxiliares. El skillmix promedio de cada hospital se obtuvo a partir de las respuestas individuales de las encuestadas en dicho hospital.

El burnout se midió con la subescala validada al español de Desgaste Emocional del Maslach Burnout Inventory ${ }^{23}$ que cuenta con 9 preguntas, cuyas respuestas se puntúan de 0 a 6 . Se consideró burnout un puntaje igual o mayor a 27 . La insatisfacción laboral se midió a través de la pregunta: “¿Cuál es tu grado de satisfacción con tu trabajo actual en este hospital?". Las posibles respuestas eran: muy insatisfecha, algo insatisfecha, moderadamente satisfecha y muy satisfecha. Se consideraron insatisfechas las enfermeras que respondieron "algo insatisfecha" o "muy insatisfecha”. La intención de dejar el trabajo se midió a través de la pregunta dicotómica: "Si pudieras, ¿dejarías este hospital a lo largo del próximo año?”.

La recolección de datos se realizó entre mayo 2017 y octubre 2018.

Para el análisis descriptivo se utilizaron frecuencias y porcentajes para variables categóricas; promedios, rangos y desviación estándar para variables continuas. Para el análisis inferencial todas las variables dependientes se dicotomizaron, utilizándose modelos de regresión logística multinivel, considerando la agrupación (clustering) de enfermeras de un mismo hospital. El burnout se modeló incluyendo las variables explicativas: características de las enfermeras (edad, formación de postítulo/postgrado) y características de los hospitales (localización, skillmix). Al modelo de insatisfacción, se sumó el burnout como variable explicativa. Al modelo de intención de dejar el trabajo se añadieron burnout e insatisfacción como variables explicativas.

Según fuese de interés, se utilizaron los hospitales o las enfermeras como unidad de análisis. Se estableció un nivel de significación de 0,05.

Para los análisis se utilizó Stata 15.1.

\section{Resultados}

Participaron 34 instituciones: 10 de Santiago, 10 del norte y 14 del sur del país (tasa de respuesta
92\%). Los tres hospitales faltantes accedieron a participar una vez cerrada la recolección de datos. Todas las regiones estuvieron representadas a excepción de Aysén. Los hospitales variaban entre 146 y 771 camas.

Respondieron el cuestionario 1.395 enfermeras (tasa de respuesta 75\%). El mayor porcentaje corresponde a mujeres jóvenes, con pocos años de experiencia. Un $27 \%$ tenía estudios de postítulo o postgrado (Tabla 1 ).

Respecto de las dotaciones, el promedio de pacientes por enfermera fue de 14 (rango 9-23), variando entre 12,4 y 15,6 al comparar turnos de día y noche (Tabla 2). La diferencia resultó estadísticamente significativa ( $\mathrm{p}<0,0001)$. El aumento en la asignación de pacientes durante la noche alcanzó un máximo de 13 pacientes en

\section{Tabla 1. Características de las Enfermeras (n = 1.395)}

\begin{tabular}{|lr|}
\hline Característica & \\
\hline Mujeres N (\%) & $1.204(86,7)$ \\
\hline Edad $\bar{x}(D S)$ & $31,9(7,4)$ \\
Mujeres menores de 30 años N (\%) & $682(48,9)$ \\
Años experiencia $\bar{x}(D S)$ & $6,2(6,9)$ \\
Especialidad N (\%) & $337(24,5)$ \\
Magíster N (\%) & $34(2,5)$ \\
\hline
\end{tabular}

n: tamaño muestral, N: número, $\bar{x}$ : promedio, DS: desviación estándar.

Tabla 2. Dotaciones y Skillmix hospitalarios ( $n=34)$

\begin{tabular}{|lcc|}
\hline & $\begin{array}{c}\text { Pro- } \\
\text { medio }\end{array}$ & Rango \\
\hline Dotaciones & & \\
Pacientes por enfermera-general & 14,1 & $9,4-23,0$ \\
Pacientes por enfermera-turno día & 12,4 & $8,7-20,1$ \\
Pacientes por enfermera-turno noche & 15,6 & $10,0-31,5$ \\
Skillmix & & \\
Porcentaje de enfermeras-general & 30,6 & $19,1-35,9$ \\
Porcentaje de enfermeras-turno día & 31,7 & $21,5-39,8$ \\
Porcentaje de enfermeras-turno noche & 29,7 & $14,3-36,4$ \\
\hline
\end{tabular}
$\mathrm{n}$ : tamaño muestral. 
un hospital, correspondiendo a $60 \%$ de aumento de la carga laboral en dicho hospital. Sobre el skillmix, 6 hospitales tenían menos de 25\% de enfermeras. Se observó una diferencia estadísticamente significativa entre el skillmix de día y de noche $(\mathrm{p}<0,0001)$ (Tabla 2). El análisis de dotación y skillmix mostró una relación inversa; en general, a mayor carga de pacientes, menor skillmix (Figura 1).

Respecto de los indicadores laborales, se observó gran variabilidad entre hospitales. El de mayor prevalencia fue el burnout, con un promedio de $34,7 \%$. La edad se asoció significativamente a burnout; las enfermeras más jóvenes presentaron más burnout. Se observó un resultado similar en las enfermeras con menos años de experiencia, aunque no se alcanzó significancia estadística. La insatisfacción fue el indicador laboral menos prevalente, y no se observó asociación con las variables de enfermería estudiadas; sin embargo, en los hombres y en las enfermeras con estudios de postítulo o postgrado se observó una frecuencia mayor de insatisfacción laboral. En cuanto a la intención de dejar el trabajo, el porcentaje fue significativamente mayor entre enfermeras con estudios de postítulo o posgrado. También hubo diferencias, aunque no fueron significativas, entre enfermeras más jóvenes y con menor tiempo de experiencia laboral (Tabla 3 ).
La matriz de correlación mostró una correlación positiva significativa entre burnout, insatisfacción e intención de dejar el trabajo $(\mathrm{p}<0,0001$ para cualquiera de las correlaciones). La correlación más fuerte se observó entre insatisfacción e intención de dejar el trabajo $(\mathrm{r}=0,44)$.

En el análisis inferencial, se observó asociación significativa entre burnout e insatisfacción laboral e intención de dejar el trabajo (Tabla 4). La edad se mostró como factor protector de burnout e intención de dejar el trabajo. Por cada año añadido a la edad de una enfermera, el riesgo de burnout decrece en 2\%. Trabajar en Santiago aumenta el riesgo de burnout e intención de dejar el trabajo. El skillmix solo se asoció significativamente a burnout; el aumento de 10 puntos porcentuales se asoció a una reducción de un $28 \%$ en la odds de burnout.

\section{Discusión}

Este es el estudio más grande sobre recurso humano de enfermería en el sistema público de salud en Chile. Revela problemáticas que no solo afectan a las enfermeras, sino que pueden menoscabar la calidad de atención. Destaca la variabilidad de dotaciones y skillmix entre hospitales. Esto refleja una falta de estandarización, pese a las recomenda-

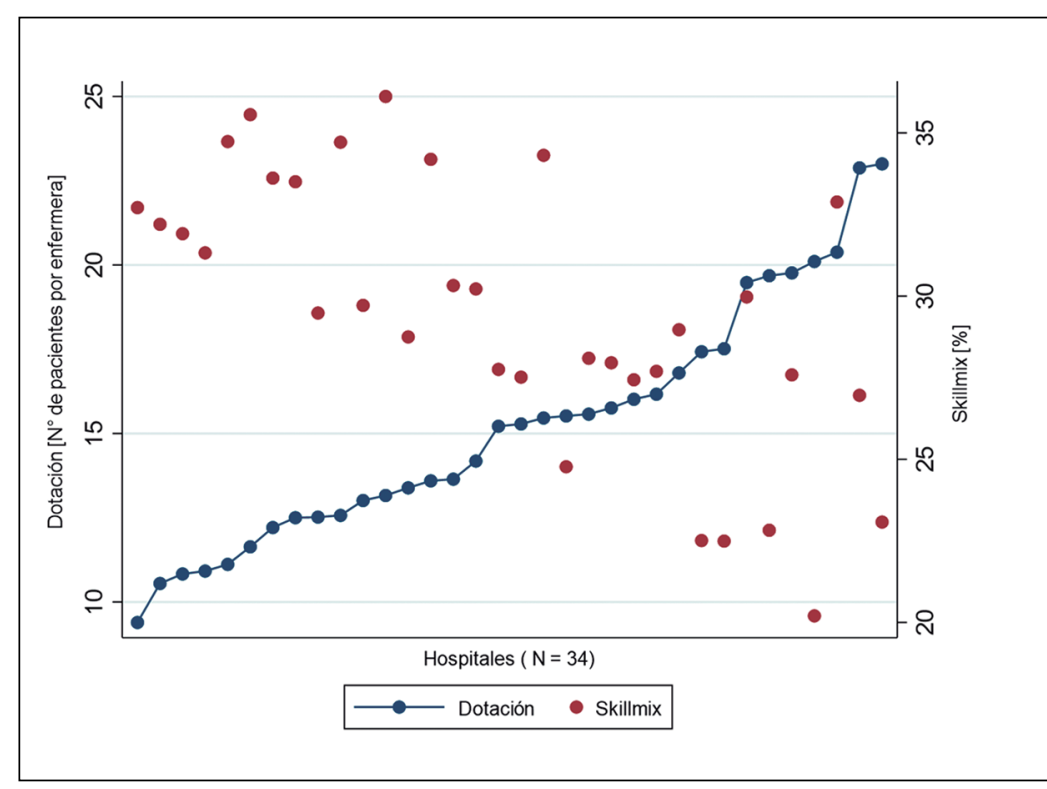

Figura 1. Relación entre Dotaciones de Enfermería y Skillmix Hospitalarios $(n=34)$. 
Tabla 3. Porcentaje de Burnout, Insatisfacción Laboral e Intención de dejar el Trabajo a nivel hospital y según características de las enfermeras

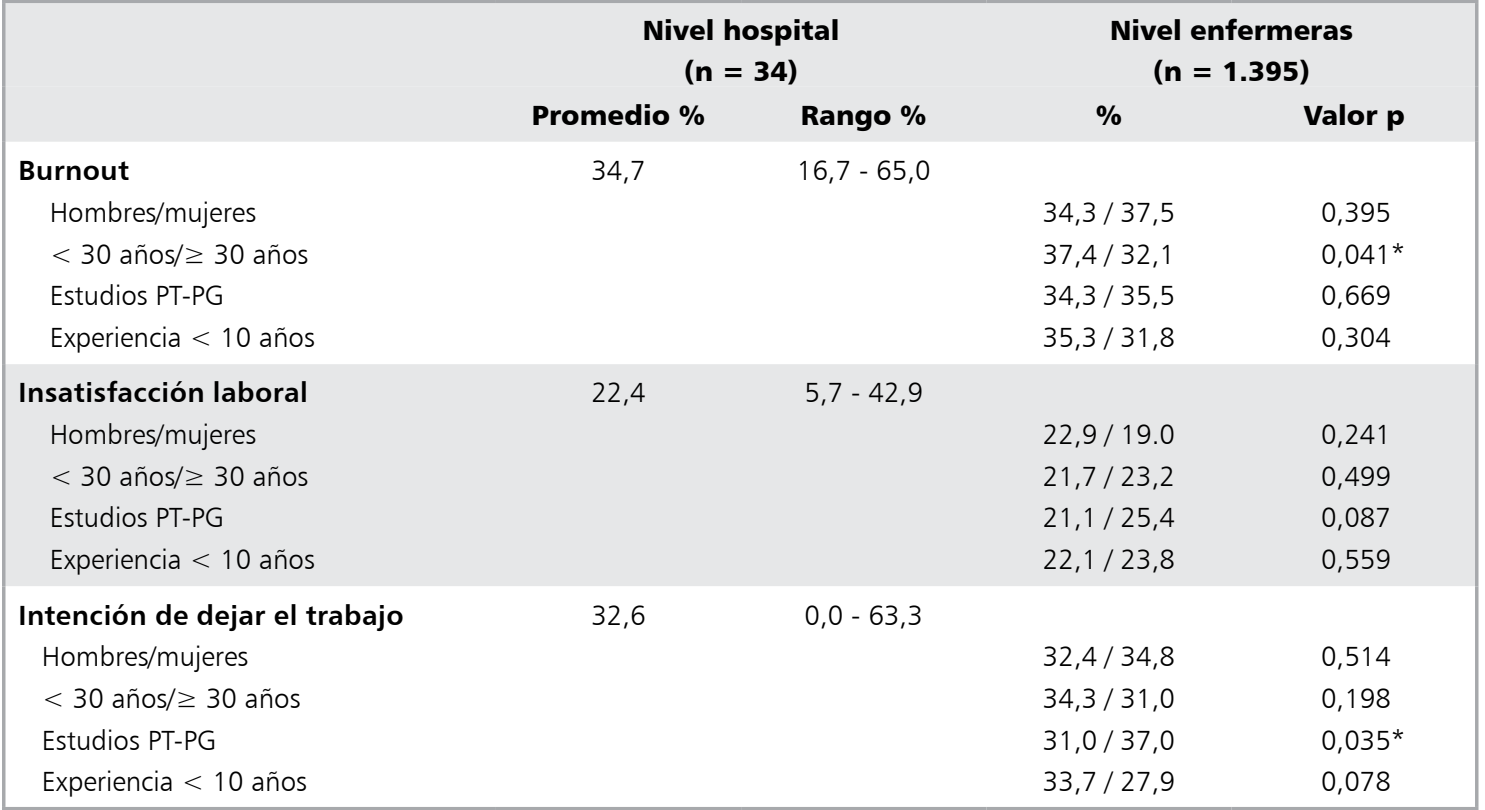

*Nivel de significación estadística $<0,05$.

Tabla 4. Asociación de variables demográficas de enfermería y de los hospitales con indicadores laborales de enfermería $(n=1.395)$

\begin{tabular}{|c|c|c|c|c|c|c|c|c|c|}
\hline & \multicolumn{3}{|c|}{ Burnout } & \multicolumn{3}{|c|}{ Insatisfacción } & \multicolumn{3}{|c|}{$\begin{array}{c}\text { Intención de dejar el } \\
\text { trabajo }\end{array}$} \\
\hline & OR & $95 \%$ IC & $\mathbf{p}$ & OR & $95 \%$ IC & $\mathbf{p}$ & OR & $95 \%$ IC & $\mathbf{p}$ \\
\hline Burnout & & & & 1,09 & $1,07-1,10$ & $<0,0001$ & 1,06 & $1,05-1,07$ & $<0,0001 *$ \\
\hline Insatisfacción & & & & & & & 5,19 & $3,78-7,14$ & $<0,0001 *$ \\
\hline Edad & 0,98 & $0,97-0,99$ & $0,003^{*}$ & 1,01 & $0,99-1,03$ & 0,229 & 0,96 & $0,94-0,98$ & $<0,0001 *$ \\
\hline Educación de postítulo/postgrado & 1,07 & $0,84-1,37$ & 0,566 & 1,18 & $0,88-1,59$ & 0,263 & 1,53 & $1,11-2,10$ & $0,0010^{*}$ \\
\hline Localización en Santiago vs provincia & 1,46 & $1,06-1,99$ & 0,019 & 0,91 & $0,65-1,30$ & 0,632 & 1,43 & $1,10-1,86$ & $0,007^{*}$ \\
\hline Skillmix ${ }^{\#}$ & 0,72 & $0,54-0,97$ & $0,029 *$ & 1,19 & $0,77-1,85$ & 0,442 & 0,91 & $0,59-1,40$ & 0,673 \\
\hline
\end{tabular}

Análisis de regresión logística, considerando clustering de enfermeras por hospital $(\alpha=0,05)$. " La interpretación de la variable skillmix considera cambios en el resultado por cada 10 puntos porcentuales de aumento en el skillmix. n: tamaño muestral, OR: odds ratio, IC: intervalo de confianza.

ciones ministeriales en la materia ${ }^{16}$, y una inequidad en la atención, ya que en algunos hospitales los pacientes cuentan con menos horas de cuidado y supervisión de enfermería que en otros.

El promedio de pacientes por enfermera es muy superior al de países como Estados Unidos o de la Unión Europea. Por ejemplo, Inglaterra, que solo cuenta con un sistema público de salud, tiene una dotación promedio de 8,8 pacientes por enfermera ${ }^{8}$. En países europeos con menor bienestar económico, como España, la dotación promedio es de $12,7^{8}$. Por otra parte, la variabilidad de do- 
tación entre día y noche hace precario el cuidado de los pacientes en turno de noche en aquellos hospitales en que ya la dotación general es deficiente. Sumado a lo anterior, el skillmix habla de que los cuidados de enfermería están brindándose en gran proporción por personal no profesional. Hay que tener en cuenta que la complejidad de los pacientes hospitalizados es cada vez mayor, por lo que la toma de decisiones requiere de un criterio profesional. Estudios en Estados Unidos de Norteamérica y Europa coinciden en que la substitución de enfermeras por personal menos cualificado pone en riesgo la salud y deteriora la experiencia de los pacientes ${ }^{24,25}$.

Los indicadores laborales de enfermería constituyen otra alerta. Alrededor de un tercio de las enfermeras tiene burnout e intención de dejar su trabajo, mientras que un quinto está insatisfecha. Estos indicadores pueden también afectar negativamente la atención. El burnout, sobre todo en contextos de sobrecarga de trabajo, disminuye la capacidad de las personas frente a las demandas de trabajo, deteriora el compromiso con la organización, aumenta el ausentismo, y puede gatillar la decisión de renunciar ${ }^{13}$. Similares consecuencias se asocian a insatisfacción laboral ${ }^{26}$. La renuncia y rotación de enfermeras agrava la subdotación y las enfermeras que permanecen en la organización sufren las consecuencias de una mayor demanda de trabajo. Además, la rotación hace que se pierda el personal de más experiencia y se reemplace por profesionales que requieren de muchas horas de formación para entregar una atención equivalente a la de sus pares. Esta situación, a su vez, aumenta los costos de personal ${ }^{27}$.

Conocer los factores que se asocian a los indicadores laborales es de valor para la gestión de los equipos de enfermería. Este estudio identificó una asociación significativa entre la edad de las enfermeras y el burnout y la intención de dejar el trabajo; hay estudios previos con similares resultados (Flinkman, Leino-Kilpi, \& Salanterä, 2010; Gómez-Urquiza, Vargas, De la Fuente, Fernández-Castillo, \& Cañadas-De la Fuente, 2017), aunque con respecto a la edad y burnout los hallazgos no han sido siempre consistentes (Pérez-Fuentes, Molero-Jurado, Gázquez-Linares, \& Simón-Márquez, 2019). El que las enfermeras jóvenes tengan mayor riesgo de burnout e intención de dejar su trabajo requiere la atención de las autoridades sanitarias, sobre todo considerando que casi el
$50 \%$ de las enfermeras son menores de 30 años. Entre las enfermeras con estudios de postítulo/ postgrado y entre aquellas que trabajan en Santiago es más frecuente la intención de dejar el trabajo. En ambos casos el factor determinante puede ser la percepción de más oportunidades laborales. La evidencia muestra que la falta de perspectivas de carrera dentro de la institución y de reconocimiento son factores asociados a mayor rotación (Flinkman et al., 2010). Las administraciones de los hospitales debieran enfrentar el desafío de retener a aquellas enfermeras que, acumulando experiencia y teniendo más estudios, están en condiciones de elevar la calidad de atención. Esto pasa por contar con incentivos, reconocimientos, y planes de carrera y desarrollo profesional.

Este estudio tiene la limitación de ser transversal, lo que no permite estudiar causalidad entre las variables de interés. Sin embargo, los datos aportados son valiosos y únicos y abren camino a futuras líneas de investigación. Respecto de la muestra de hospitales, los tres que no participaron en el estudio son de la misma región, que podría haber quedado menos representada. Aun así, no hay razones para creer que dichos hospitales difieren de manera significativa de los estudiados y que su inclusión hubiese cambiado los resultados del estudio.

Los resultados de este estudio son una llamada a atender a la problemática de las dotaciones, el burnout y la rotación de enfermeras en los hospitales chilenos. El siguiente paso es analizar cómo dichos factores afectan los resultados clínicos y la experiencia de los pacientes. Entender estas asociaciones puede entregar a las autoridades sanitarias evidencia valiosa para elaborar políticas de salud que consideren la contribución de enfermería al mejoramiento de la calidad.

Agradecimientos: Agradecemos a los profesores Douglas Sloane, Jere Behrman, Herbert Smith y al analista de datos Tim Cheney, de la Universidad de Pennsylvania. También agradecemos a quienes contribuyeron de forma importante en la recolección de datos del estudio: Ana María Vásquez, profesora de la Escuela de Enfermería de la Universidad de La Serena; Dina Alfaro, Yessica Benavides, Katherinne Estrada, Karen González, Paulina Letelier, Flérida Rivera, Marina Saavedra, Leyla Sáez, Marisol Salgado, profesoras de la Escuela de Enfermería de la Universidad Católica del Maule. 


\section{Referencias}

1. Ministerio de Salud de Chile [Internet]. Programa de Salud 2018-2022. Disponible en: http://www.minsal.cl/ programa-de-salud-2018-2022/

2. Ministerio de Salud de Chile [Internet]. Informe sobre brechas de personal de salud por Servicio de Salud. 2017. Disponible en: http://web.minsal.cl/wp-content/ uploads/2015/08/Informe-Brechas-RHS-en-Sector-Público_Marzo2016.pdf

3. Buchan J, O’May F, Dussault G. Nursing workforce policy and the economic crisis: A global overview. J Nurs Scholarsh 2013; 45 (3): 298-307.

4. Shin S, Park JH, Bae SH. Nurse staffing and nurse outcomes: A systematic review and meta-analysis. Nurs Outlook 2018; 66 (3): 273-82.

5. Aiken LH, Sloane D, Griffiths P, Rafferty AM, Bruyneel L, McHugh M, et al. Nursing skill mix in European hospitals: Cross-sectional study of the association with mortality, patient ratings, and quality of care. BMJ Qual Saf 2017; 26 (7): 559-68.

6. Lake ET, Sanders J, Duan R, Riman KA, Schoenauer $\mathrm{KM}$, Chen Y. A meta-analysis of the associations between the nurse work environment in hospitals and 4 sets of outcomes. Med Care 2019; 57 (5): 353-61.

7. Aiken LH, Sloane DM, Ball J, Bruyneel L, Rafferty A M, Griffiths P. Patient satisfaction with hospital care and nurses in England: An observational study. BMJ Open 2018; 8 (1): 1-8.

8. Aiken LH, Sloane DM, Bruyneel L, Van Den Heede K, Griffiths P, Busse R, et al. Nurse staffing and education and hospital mortality in nine European countries: A retrospective observational study. The Lancet 2014; 383 (9931): 1824-30.

9. Shekelle PG. Nurse-patient ratios as a patient safety strategy. Ann Intern Med 2014; 158 (5): 417-26.

10. Nantsupawat A, Nantsupawat R, Kunaviktikul W, Turale S, Poghosyan L. Nurse Burnout, Nurse-Reported Quality of Care, and Patient Outcomes in Thai Hospitals. J Nurs Scholarsh 2016; 48 (1): 83-90.

11. Perry SJ, Richter JP, Beauvais B. The effects of nursing satisfaction and turnover cognitions on patient attitudes and outcomes: A three-level multisource study. Health Serv Res 2018; 53 (6): 4943-69.

12. Halter M, Boiko O, Pelone F, Beighton C, Harris R, Gale $\mathrm{J}$, et al. The determinants and consequences of adult nursing staff turnover: A systematic review of systematic reviews. BMC Health Serv Res 2017; 17 (1): 824.

13. Maslach C, Leiter MP. Understanding the burnout experience: Recent research and its implications for psychiatry. World Psychiatry 2016; 15 (2): 103-11.
14. Maslach C, Leiter MP. New insights into burnout and health care: Strategies for improving civility and alleviating burnout. Medical Teacher 2017; 39 (2): 160-3.

15. Vergara M. Propuesta de reformas a los prestadores públicos de servicios médicos en Chile: Fortaleciendo la opción pública. Rev Med Chile 2015; 143 (2): 23743.

16. Ministerio de Salud de Chile. Recomendación sobre Estándares de Dotación en Establecimientos de Atención Cerrada. 2012.

17. Aiken LH, Sermeus W, Van Den Heede K, Sloane DM, Busse R, McKee M, et al. Patient safety, satisfaction, and quality of hospital care: Cross sectional surveys of nurses and patients in 12 countries in Europe and the United States. BMJ 2012; 344 (7851): 1-14.

18. Palma FS, Suazo SV. Síndrome de burnout en trabajadores de enfermería de dos hospitales del sur de Chile. Av Enferm 2016; 34 (1): 39-47.

19. Soto P, Barrios S, Molina Y. Síndrome de quemarse por el trabajo y satisfacción laboral como predictores de calidad de la atención de enfermería hospitalaria. Cienc Enferm 2017; (3): 99-111.

20. Dirección de Presupuestos Gobierno de Chile. Anuario estadístico del empleo público en el gobierno central 2011-2018. 2019. Disponible en: http://www.dipres. cl/598/articles-191413_doc_pdf.pdf

21. Sermeus W, Aiken LH, Van Den Heede K, Rafferty AM, Griffiths P, Moreno MT, et al. Nurse forecasting in Europe (RN4CAST): Rationale, design and methodology. BMC Nurs 2011; 10 (1): 6.

22. Aiken LH, Cerón MC, Simonetti M, Lake ET, Galiano A, Garbarini, A, et al. Hospital nurse staffing and patient outcomes. Rev Med Clín Condes 2018; 29 (3): 322-7.

23. Olivares VE, Mena L, Jelvez C, Macía F. Validez factorial del Maslach Burnout Inventory human services (MBI-HSS) en profesionales chilenos. Universitas Psychologica 2014; 13 (1): 145-60.

24. Aiken LH, Sloane D, Griffiths P, Rafferty AM, Bruyneel L, McHugh M, et al. Nursing skill mix in European hospitals: Cross-sectional study of the association with mortality, patient ratings, and quality of care. BMJ Qual Saf 2017; 26 (7): 559-68.

25. Needleman J. Nursing skill mix and patient outcomes. BMJ Qual Saf 2017; 26 (7): 525-8.

26. Lu H, Zhao Y, While AE. Job satisfaction among hospital nurses: A literature review. Int J of Nurs Stud 2019; 94 (2): 21-31.

27. Kovner CT, Brewer CS, Fatehi F, Jun J. What does nurse turnover rate mean and what is the rate? Policy, Politics, and Nursing Practice 2014; 15 (3-4): 64-71. 
28. Flinkman, M, Leino-Kilpi H, \& Salanterä S. Nurses' intention to leave the profession: Integrative review. J Adv Nurs 2010; 66 (7): 1422-34.

29. Gómez-Urquiza JL, Vargas C, De la Fuente EI, Fernández-Castillo R, \& Cañadas-De la Fuente GA. Age as a risk factor for burnout syndrome in nursing professio- nals: A meta-analytic study. Res Nurs Health 2017; 40 (2): 99-110.

30. Pérez-Fuentes M, Molero-Jurado M, Gázquez-Linares JJ, \& Simón-Márquez M. Analysis of burnout predictors in nursing: Risk and protective psychological factors. Eur. J Psychol Appl to Leg Context 2019; 11 (1): 33-40. 